\title{
Squamous Cell Carcinoma Arising from Sinonasal Inverted Papilloma
}

\author{
(DD.T. Ginat, (D) A. Trzcinska, and (DP. Horowitz
}

\begin{abstract}
SUMMARY: Sinonasal inverted papillomas occasionally undergo malignant transformation into squamous cell carcinoma, which can be associated with EGFR mutations. Since biopsy can potentially under-sample the tumor, CT and MRI can provide clues as to the presence of malignant transformation. In particular, this entity tends to appear different from benign inverted papilloma on imaging, including prominent bone erosions, necrosis, low diffusivity in the solid tumor components, and absence of the cerebriform pattern on MRI. The radiology findings, pathology features, and management of squamous cell carcinoma arising from inverted papilloma are described.
\end{abstract}

ABBREVIATION: EGFR = epidermal growth factor receptor

$\mathbf{T}$ he patient is a 61-year-old male former 40 pack-year smoker who presented with headaches and discharge of material from his nose for the past 9 months. The patient also experienced diplopia with left lateral gaze and tongue deviation to the left. Examination revealed a sinonasal mass that appeared pinkish-red with a centrally necrotic, pearly-white core. The mass was biopsied at an outside hospital and interpreted as inverted papilloma. The patient was then referred to our institution for further management.

\section{IMAGING}

CT showed diffuse opacification of the sphenoid sinuses, posterior ethmoid sinuses, and posterior nasal cavity with a relatively well-defined mass with peripheral enhancing components and central heterogeneous nonenhancing components, as well as associated extensive remodeling and erosion of the sphenoid sinus walls, floor of the sella, and clivus, including the left hypoglossal canal, which accounted for the tongue weakness (Fig 1). MR imaging demonstrated a large peripherally enhancing mass that appeared to potentially invade the bilateral cavernous sinuses and sella without a bone marrow replacement process beyond the mass (Fig 2). In particular, there was rather thin diffuse peripheral enhancement of the tumor with relatively low diffusivity and

Received March 4, 2020; accepted after revision March 26.

From the Departments of Radiology, Section of Neuroradiology (D.T.G.), Pathology (A.T.), and Surgery, Section of Neurosurgery (P.H.), University of Chicago, Chicago, Illinois.

Please address correspondence to Daniel Thomas Ginat, MD, MS, University of Chicago, 5841 S Maryland Ave, Chicago, IL 60637; e-mail: dtg1@uchicago.edu

http://dx.doi.org/10.3174/ajnr.A6583 central areas of heterogeneous T2 signal, indicative of necrosis with relatively high diffusivity. Based on the atypical imaging findings for benign inverted papilloma, the possibility of malignant transformation was suspected.

\section{Treatment}

Endoscopic endonasal resection was performed, which showed viable tumor peripherally, with extensive central necrosis. The bulk of the tumor was removed with a combination of suction, debriding, and grasping instruments. The sella and right cavernous sinus were displaced peripherally, with good planes of separation and negative pathologic margins on the overlying mucosa. However, positive microscopic margins were noted along the left cavernous sinus wall, left sphenoid roof, and sphenoid floor, from which the mass appeared to be arising. The tumor also invaded the clivus, upper pharyngeal musculature, and left hypoglossal canal, which was decompressed but contained invasive gross tumor. These findings correlated well with the imaging findings (Fig 3 ), specifically with regard to areas of necrosis and viable tumor, though we were pleasantly surprised by the lack of invasion along the right side of the skull base. The resulting defect was repaired with a fascia lata graft and nasoseptal flaps, to provide healthy tissue covering the carotid arteries in preparation for planned adjuvant radiation therapy and weekly cisplatin. Postoperatively, the patient recovered well and experienced improved tongue mobility, resolution of the diplopia, and decreased headaches.

\section{Diagnosis}

Intraoperative frozen sections revealed squamous cell carcinoma arising from sinonasal papilloma. Histologic sections showed a 

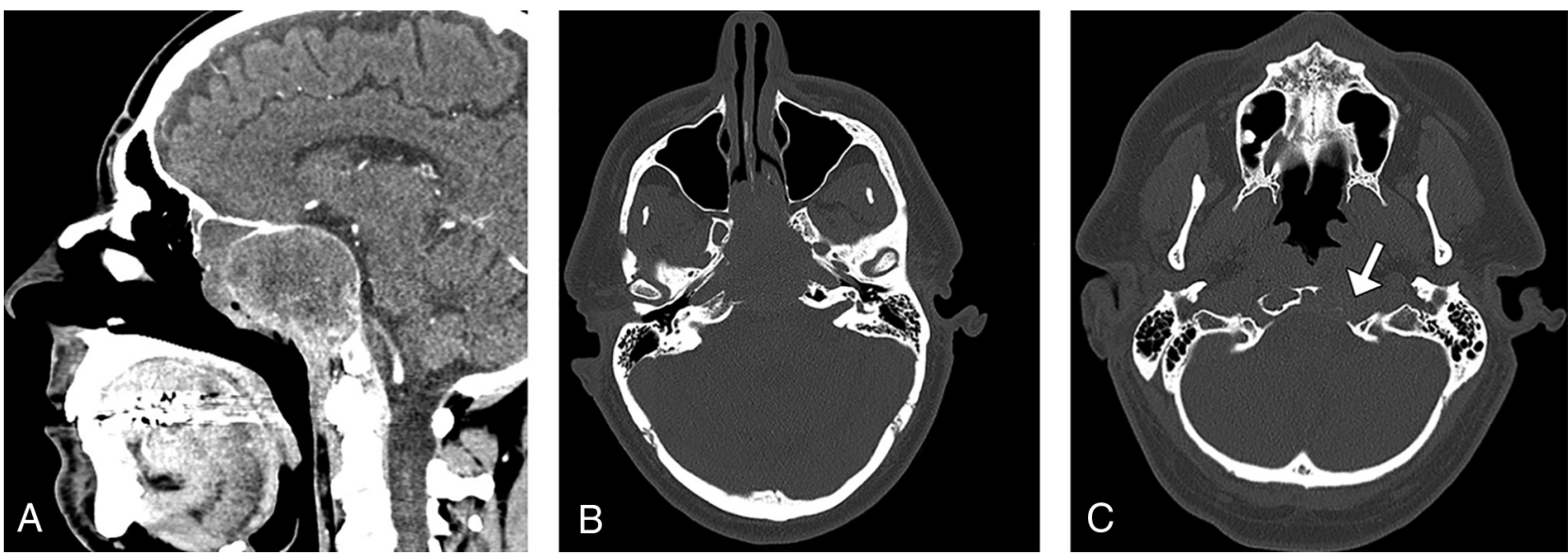

FIG 1. Sagittal postcontrast CT image $(A)$ shows a fairly well-defined-but-heterogeneous mass in the posterior nasal cavity and ethmoid and sphenoid sinuses, with peripheral areas of enhancement and mixed areas of central hypoattenuation and hyperattenuation. Axial CT images in bone windows ( $B$ and $C$ ) show extensive erosion of the sinus walls and clivus, with involvement of the left hypoglossal canal (arrow).
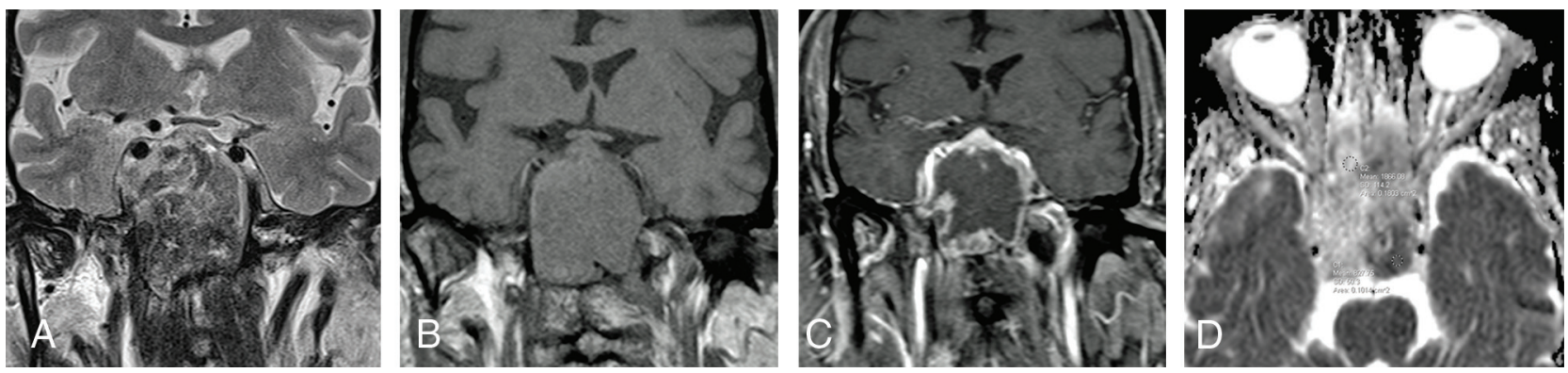

FIG 2. Coronal T2-weighted $(A)$ MR imaging shows a heterogeneous mass occupying the sphenoid sinuses with extension into the cavernous sinuses and sella. The T1-weighted $(B)$ and postcontrast T1-weighted $(C)$ MR images show diffuse peripheral enhancement. The ADC map (D) shows relatively high diffusivity in the necrotic portions of the tumor and low diffusivity within the viable portions of the tumor.

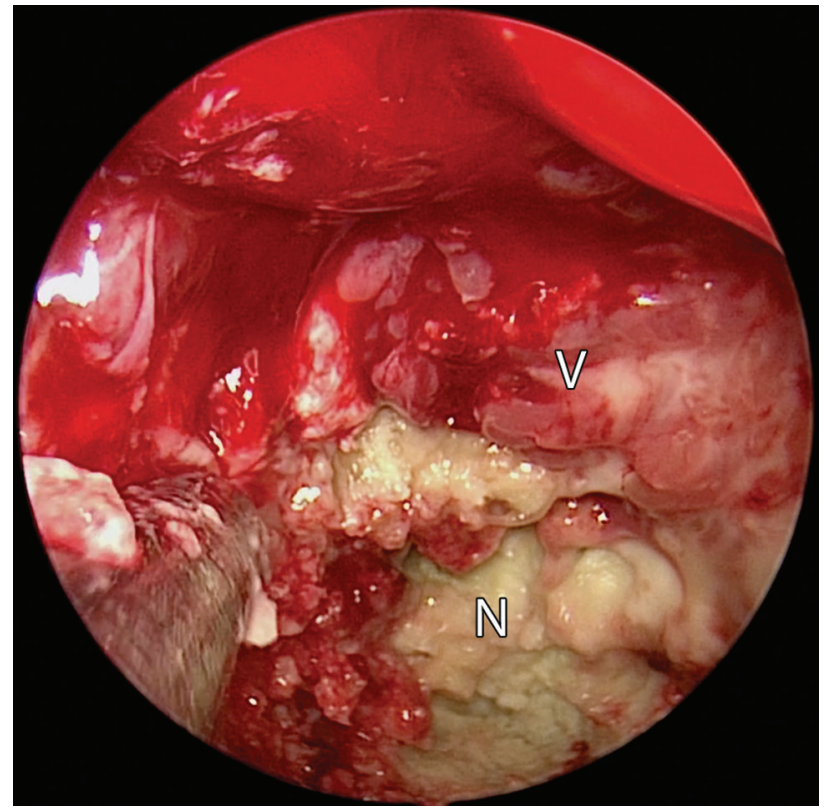

FIG 3. Endoscopic view during endonasal surgery shows viable $(\mathrm{V})$ and necrotic $(\mathrm{N})$ tumor components. sinus lesion exhibiting an inverted growth pattern typical of a sinonasal papilloma, inverted type (Fig 4). The appearance of the lesional epithelium varied from conventional (acanthotic transitional-like epithelium with ciliated surface cells and intraepithelial neutrophilic microabscesses) to dysplastic (squamous metaplasia with nuclear enlargement, nuclear membrane irregularity, hyperchromasia, loss of polarity, and increased mitotic activity). Rare foci of superficial invasion into the underlying stroma and extensive areas of necrosis were also present. Thus, the presence of a conventional inverted papilloma, together with extensive dysplasia, foci of invasive carcinoma, and, in particular, the extensive necrosis matched the clinicoradiologic suspicion. DNA was also isolated from the specimen, which revealed an epidermal growth factor receptor (EGFR) mutation involving a 9-base pair duplication in exon 20, along with several other alterations of uncertain significance.

\section{DISCUSSION}

Inverted papilloma is a benign but locally aggressive sinonasal neoplasm. This lesion can be associated with squamous cell carcinoma in approximately $7 \%-16 \%$ of cases, either synchronously or metachronously. ${ }^{1-3}$ Although inverted papillomas with malignant degeneration are most commonly located in the 

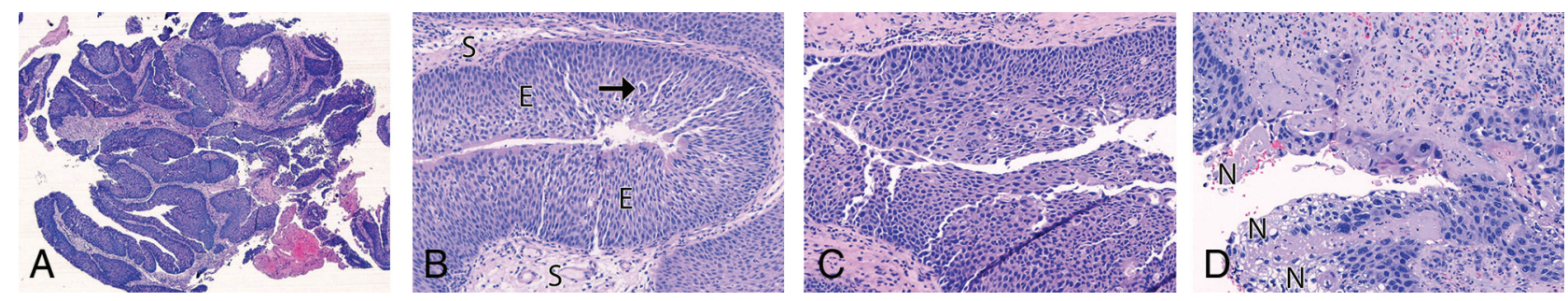

FIG 4. Hematoxylin-eosin-stained photomicrographs of the lesion show an inverted growth pattern from the sinonasal papilloma component ( $A, \times 4$ magnification), markedly thickened epithelium (E) with ciliated luminal cells and intraepithelial neutrophilic microcysts (arrow) growing downward into the underlying stroma $(S)(B, \times 20$ magnification), areas of severe epithelial dysplasia ( $C, \times 20$ magnification), and foci of superficial invasion and tumor necrosis with apoptotic cells $(N)(D, \times 20$ magnification).

lateral nasal cavity or maxillary sinus, tumors arising in the frontal sinus or the frontoethmoidal recess are proportionately most likely to be associated with carcinoma. ${ }^{2}$ Sphenoid sinus involvement, such as in this case, is relatively uncommon for both benign inverted papillomas and those associated with squamous cell carcinoma.

Various risk factors are implicated in the malignant transformation of sinonasal papillomas, including human papillomavirus infection, smoking, occupational exposure, and DNA alterations, such as EGFR mutations. ${ }^{4,5}$ Of note, EGFR is the gene that encodes the epidermal growth factor receptor, which is a cell membrane protein that is involved in tyrosine phosphorylation and cell proliferation. The specificity of EGFR mutations suggests that squamous cell carcinoma arising from the inverted papilloma disease spectrum is biologically distinct from other sinonasal squamous lesions. ${ }^{6}$

Distinguishing benign inverted papilloma and inverted papilloma associated with malignant transformation can be challenging clinically. Both entities can present with nasal obstruction and rhinorrhea, but epistaxis, vision changes, and pain are ominous signs suggestive of malignancy. ${ }^{3,7}$ As in this case, the diagnosis is sometimes missed by biopsy because tissue containing malignant cells is not always obtained. ${ }^{3}$ However, imaging with $\mathrm{CT}$ and MR imaging can help characterize these tumors in terms of identifying atypical features and delineating the extent of tumor for guiding further management.

Benign sinonasal inverted papillomas characteristically demonstrate osteitis at the attachment site on CT and a convoluted cerebriform pattern consisting of alternating bands of high and low signal on T2-weighted and postcontrast T1-weighted MR imaging sequences. ${ }^{8}$ On the other hand, inverted papillomas with malignant transformation tend to have imaging characteristics distinct from those of benign inverted papillomas. In particular, the presence of bone erosions on CT is suggestive of malignancy, though the margins of the tumor can be relatively sharp, with bone remodeling suggestive of a more indolent process as in this case. On MR imaging, the absence of the classic cerebriform or columnar pattern associated with inverted papilloma and the presence of necrotic components are indicative of squamous cell carcinoma. ${ }^{9,10}$ In addition, the viable portions of tumors with malignant transformation tend to have significantly lower ADC values than benign inverted papillomas, with an average diffusion coefficient of $1.1 \times 10^{-3} \mathrm{~mm}^{2} / \mathrm{s}$ versus of $1.5 \times 10^{-3} \mathrm{~mm}^{2} / \mathrm{s}$. However, the presence of bulk necrosis in tumors is generally associated with elevated diffusivity. ${ }^{11}$
Aggressive surgical resection combined with postoperative adjuvant therapy, including chemotherapy and radiation, can be effective in managing even stage T4 squamous cell carcinomas arising from inverted papillomas. ${ }^{12}$ The goal of surgery is obtaining negative microscopic margins, though with tumor invasion of critical skull base structures, this may not be possible in some cases. The anatomy of the tumor generally dictates the approach: Depending on the extent of disease, endoscopic sinus surgery alone, endoscopic sinus surgery plus endonasal or open anterior maxillotomy, and endoscopic sinus surgery plus an anterior craniotomy can be considered. ${ }^{12}$ In this case, the tumor likely originated from the left sphenoid sinus, while growing displaced the cavernous sinuses, sella, and other structures outward, allowing a wide surgical corridor by an endoscopic endonasal approach simply by debriding the necrotic core. Because patients with sinonasal malignancies typically require adjuvant chemoradiation, repair of the skull base defect is an important consideration. Specifically, exposed neurovascular structures, such as the carotid arteries and cavernous sinuses, should be covered with healthy, preferably vascularized tissue or flaps, to the reduce risk of osteoradionecrosis or carotid blowout.

The prognosis of patients with inverted papillomas associated with squamous cell carcinoma depends largely on the tumor stage, with an overall 3-year disease-specific survival rate of $63 \%{ }^{3}$ Most tumor recurrences are attributable to incomplete resection, and close life-long imaging follow-up is warranted, with biopsies performed depending on the imaging findings. ${ }^{1}$ In certain cases with $E G F R$ mutations, irreversible tyrosine kinase inhibitors can result in inactivation of EGFR signaling and growth inhibition. ${ }^{5}$ However, there is evidence that most exon 20 insertion mutations confer resistance to EGFR tyrosine kinase inhibitor therapy, at least in lung cancer. ${ }^{13}$

\section{Case Summary}

- It is not uncommon for sinonasal inverted papilloma to undergo malignant transformation into squamous cell carcinoma, which can be associated with EGFR mutations, as in this case.

- Because biopsy can undersample the lesion, the presence of aggressive features on imaging should raise the possibility of malignant transformation.

- The presence of necrosis and low diffusivity instead of a cerebriform appearance on MRI and extensive bone erosions demonstrated on CT are suggestive of associated squamous cell carcinoma. 
- CT and MRI are useful for tumor localization and treatmentplanning, which generally consists of surgical resection, chemotherapy, and radiation for inverted papillomas with malignant transformation.

- ADC can be useful for mapping heterogeneous tumors, with low diffusivity associated with viable portions of malignant neoplasm versus high diffusivity associated with bulk necrosis.

\section{REFERENCES}

1. von Buchwald C, Bradley PJ. Risks of malignancy in inverted papilloma of the nose and paranasal sinuses. Curr Opin Otolaryngol Head Neck Surg 2007;15:95-98 CrossRef Medline

2. Kim K, Kim D, Koo Y, et al. Sinonasal carcinoma associated with inverted papilloma: a report of $\mathbf{1 6}$ cases. J Craniomaxillofac Surg 2012;40:e125-29 CrossRef Medline

3. Yasumatsu R, Nakashima T, Sato M, et al. Clinical management of squamous cell carcinoma associated with sinonasal inverted papilloma. Auris Nasus Larynx 2017;44:98-103 CrossRef Medline

4. Sahnane N, Ottini G, Turri-Zanoni M, et al. Comprehensive analysis of HPV infection, EGFR exon 20 mutations and LINE1 hypomethylation as risk factors for malignant transformation of sinonasal-inverted papilloma to squamous cell carcinoma. Int J Cancer 2019;144:1313-20 CrossRef Medline

5. Udager AM, McHugh JB, Goudsmit CM, et al. Human papillomavirus (HPV) and somatic EGFR mutations are essential, mutually exclusive oncogenic mechanisms for inverted sinonasal papillomas and associated sinonasal squamous cell carcinomas. Ann Oncol 2018;29:466-71 CrossRef Medline

6. Udager AM, Rolland DC, McHugh JB, et al. High-frequency targetable EGFR mutations in sinonasal squamous cell carcinomas arising from inverted sinonasal papilloma. Cancer Res 2015;75:2600-06 CrossRef Medline

7. Miyazaki T, Haku Y, Yoshizawa A, et al. Clinical features of nasal and sinonasal inverted papilloma associated with malignancy. Auris Nasus Larynx 2018;45:1014-19 CrossRef Medline

8. Fang G, Lou H, Yu W, et al. Prediction of the originating site of sinonasal inverted papilloma by preoperative magnetic resonance imaging and computed tomography. Int Forum Allergy Rhinol 2016;6:1221-28 CrossRef Medline

9. Yan $\mathrm{CH}$, Tong $\mathrm{CC}$, Penta $\mathrm{M}$, et al. Imaging predictors for malignant transformation of inverted papilloma. Laryngoscope 2019;129:77782 CrossRef Medline

10. Maroldi R, Farina D, Palvarini L, et al. Magnetic resonance imaging findings of inverted papilloma: differential diagnosis with malignant sinonasal tumors. Am J Rhinol 2004;18:305-10 CrossRef

11. Maeda M, Maier SE. Usefulness of diffusion-weighted imaging and the apparent diffusion coefficient in the assessment of head and neck tumors. J Neuroradiol 2008;35:71-78 CrossRef Medline

12. Li W, Lu H, Zhang H, et al. Squamous cell carcinoma associated with inverted papilloma: recurrence and prognostic factors. Oncol Lett 2020;19:1082-88 CrossRef Medline

13. Yasuda H, Kobayashi S, Costa DB. EGFR exon 20 insertion mutations in non-small-cell lung cancer: preclinical data and clinical implications. Lancet Oncol 2012;13:e23-31 CrossRef Medline 